\title{
MedienPädagogik
}

Zeitschrift für Theorie und Praxis der Medienbildung

Jahrbuch Medienpädagogik 3.

Zweitveröffentlichung aus: Jahrbuch Medienpädagogik 3. (2003) Opladen: Leske+ Budrich. Herausgegeben von Ben Bachmair, Peter Diepold und Claudia de Witt.

\section{PISA auch für die Medienpädagogik? \\ Warum empirische Studien zur Medienkompetenz Not tun}

\author{
Hans-Dieter Kübler
}

\section{Zirkuläre Zielhorizonte}

Wieder einmal - man zählt schon gar nicht das wievielte Mal - wurde auf einem pompösen Kongress Medienkompetenz beschworen, der allseitige Einsatz für sie bzw. sie als unbedingte Voraussetzung für alle Individuen und Organisationen, die in der heraufziehenden „Informationsgesellschaft“ bestehen und die Informationstechnologien wie die neuen Medien in Bildung, Politik und Arbeitswelt umfassend nutzen wollen. Diesmal waren es die Bertelsmann Stiftung und die AOL Time Warner Foundation, die diese Forderungen auf dem „21st Century Literacy Summit“ am 7. und 8. März 2002 zu Berlin in einem umfangreichen Kommunique (www.bertelsmann-stiftung.de/documents/white_ paperdeutsch.pdf) vortrugen. Und wieder einmal stimmten 300 prominente Vertreter aus Wirtschaft, Politik, Medien, Wissenschaft und Bildung uneingeschränkt zu; erneut bestätigte sich, dass ohne Medienkompetenz kein „privates und berufliches Vorankommen in einer vernetzten Welt“ möglich sei. Für die Bildung wurde abermals postuliert, dass Medienkompetenz als vierte Kulturtechnik zu Lesen, Schreiben und Rechnen hinzukommen muss. Damit sei nicht nur das Bedienen von Computern gemeint; in Zukunft werde vor allem die Fähigkeit entscheidend sein, Informationen suchen, bewerten und produktiv nutzen zu können - genau dies werde aber an Schulen und Universitäten noch zu sehr vernachlässigt.“

Auch das von den Bildungsministerien des Bundes und der Länder 1999 einberufene .Forum Bildung" befasste sich in einer Studie, in Workshops und Expertisen mit dem schillernden Zielkomplex der Medienkompetenz (Schiersmann u.a. 2002). Während die Studie noch eingangs registrierte, dass insbesondere die „Inhalte“ der Medienkompetenz „klärungsbedürftig“ sind, zumal zwischen einer eher pädagogischen und einer eher technischinformatikorientierten Ausrichtung (ebd., S. 19), verkürzt sie das am Ende verabschiedete „Resümee“ (ebd., S. 62ff) wieder auf weithin instrumentell-technische Fertigkeiten. Zwar lässt es die beigefügten Dimensionen nach dem Was? Wozu? und Wie? inhaltlich offen, aber füllt sie auch nicht aus. Denn Medienkompetenz definiert sich aus den „sich ergänzenden Bausteinen“: „Kompetenz zur Handhabung und Nutzung von (Medien-, IuK-)Technik, 
Kompetenz zur Gestaltung von sozio-technischen Systemen mit Hilfe von (Medien-, IuK-)Technik, Kompetenz zur kundigen Kritik von (Medien-, IuK-)Technik“ (ebd., S. $64)$.

Irritierend, wenn nicht verräterisch ist an dieser Umschreibung schon, dass Medien auf Technik reduziert und ihnen kommunikative, symbolische, politische und ästhetische Potenziale mindestens explizit nicht zugesprochen werden. Just diese Perspektive impliziert Medienkompetenz indes ihrer Genese. Entsprechend ist auch die angefügte Kritikdimension mehr als oberflächlich oder nur vorgeschoben; denn Technik kritisiert man anders, eben in ihren materiellen Leistungen oder faktischen Wirkungen, aber nicht primär in ihrer symbolisch-kommunikativen oder ideologischen Bedeutung. Diese ergibt sich aus dem kollektiven Konstrukt von Technik, der Technologie, nicht aus der Technik selbst.

Ratlos lässt einen auch die Broschüre der Gesellschaft für Medienpädagogik und Kommunikationskultur (GMK) (Palme/Basis 2001), herausgegeben im Auftrag und mit Unterstützung des Bundesministeriums für Familie, Senioren, Frauen und Jugend: Ein „zeitgemäßes update“ brauche die Medienkompetenz für die sich „etablierende Wissensgesellschaft", tönt einer der Herausgeber großspurig (Palme 2001, S. 13). Zwar seien die von Dieter Baacke formulierten „grundsätzlichen Ziele und Aufgaben“ ein „solides Fundament“, aber die „sich ändernden alltäglichen Gegebenheiten“ erfordern „eine kontinuierliche zeitgemäße Ausrichtung“. Nur worin diese über die längst bekannten Ziele, aber auch über vermeintlich schicke Platituden hinaus besteht, können die unter „Grundlagen“ rubrizierten Beiträge nicht explizieren. Immerhin veranschaulichen die Projektberichte einige konkrete Optionen und Erfahrungen, können sie aber weder verallgemeinern noch systematisieren.

So scheint es auch dieses Mal wieder - und nun schon über Jahre hinweg - beim wenig erfreulichen Befund beschieden zu bleiben, dass sich unter Medienkompetenz alles - will heißen: alle erdenklichen und tatsächlich oder vermeintlich zeitgemäßen Fähigkeiten - oder auch trotz vieler Rhetorik fast nichts Definitives und Konkretes subsumieren lässt (Kübler 1999, S. 38; Schiersmann u.a. 2002, S. 51). Am Ende obsiegen allerdings jeweils die gerade marktgängigen Mediengeräte und die Anforderungen ihrer Bedienung und Nutzung sowie die Konjunkturen des Marktes insgesamt, vom Arbeitsmarkt bis zum Konsummarkt, von den Optionen der Hardware bis hin zu denen der omnipräsenten Software. Dass just diese ihre Gegenständlichkeit und damit die kognitiven Herausforderungen ihrer Bedienbarkeit sukzessive verlieren, in alltäglichen Gebrauchsgeräten integral verschwinden bzw. den menschlichen Gepflogenheiten durch mündliche Spracherkennung, alltägliche Aufgabenstrukturierung und Abbau eigener Logiken ähnlicher werden, wird bislang noch wenig beachtet. Dadurch dürften sich jedenfalls die speziellen Zielsetzungen, die sich etwa in Begrifflichkeiten wie Computerbildung oder Medienkompetenz niederschlagen, mehr und mehr erübrigen, an ihre Stelle treten wieder universelle Perspektiven, wie sie sich mit der Betonung 
von Wissensstrukturierung und -bewertung oder der zunehmenden Akzentuierung des Content (wie es neudeutsch heißt) abzeichnen.

\section{Reale Ernüchterungen und ihre medialen Sündenböcke}

Dabei haben die in der Öffentlichkeit aufgeregt aufgenommenen Ergebnisse der sogenannten PISA-Studie (Programme for International Student Assessment) der Organisation für wirtschaftliche Zusammenarbeit und Entwicklung (OECD) über das relativ schlechte Abschneiden deutscher Schülerinnen und Schüler im Alter von 15 Jahren vor allem in der Erfassung und Erschließung von Texten im Vergleich mit denen von 31 anderen Ländern Ende 2001 noch eine andere, viel gravierendere Lektion erteilt: nämlich die, dass sich zwischen obligatorischer Sollensrhetorik und Zielverbalität einerseits, schulischer Wirklichkeit und individuellen Fähigkeiten andererseits erhebliche, wenn nicht schmerzliche Diskrepanzen auftun können und dass eine Pädagogik, die sich nur in wohlklingenden Zielformulierungen und Normdefinitionen ergeht, ihre Beziehung zur Realität und damit auch ihre Legitimität als Wissenschaft verliert. Vermutlich deshalb saß der Schock so tief, war das Erschrecken so groß, und gelobten alle Beteiligten, die Politiker wie die Wissenschaftler, Besserung. Denn für praktizierende Insider, für Lehrerinnen und Lehrer, waren die Erkenntnisse weithin nicht so unerwartet, jedenfalls nicht so fürchterlich erschütternd wie für die aufgebrachte Öffentlichkeit, mindestens tendenziell und exemplarisch verzeichnen sie täglich ähnliche Erfahrungen. Allenfalls die mächtige Repräsentativität und die Wucht der komparativen Daten bestätigten sie nachdrücklich.

Als deskriptive Momentaufnahme individueller Leistungen kann PISA keine systematische Ursachenforschung betreiben, die wenigen, nur oberflächlichen Hinweise lassen sich recht ambivalent oder jeweils politisch opportunistisch interpretieren. So vernahm man etwa kaum Stimmen - von wissenschaftlicher Seite überhaupt keine -, die Zusammenhänge zwischen der Mediennutzung und -begeisterung Jugendlicher und ihren schulischen Leistungen vermuteten, obwohl solche Spekulationen sonst schnell bei der Hand sind.

Allein politisch argumentierende Protagonisten wussten offenbar sogleich Bescheid und identifizierten die Verursacher: So macht die baden-württembergische Kultusministerin Schavan den Computer als Schuldigen aus - den sonst gerade konservative Bildungspolitiker mit Macht in die Schulen hineinbugsieren und als optimales Vehikel moderner Bildung und Wissensvermittlung bejubeln. „Viele Schüler sitzen heute jeden Tag mehrere Stunden vor dem Bildschirm“, klagt die Christdemokratin gegen ihre eigene Politik. Die Mädchen und Jungen seien geprägt durch kurze und vielfältige Reize des Fernsehens und des Computers. Während die jungen Leute früher ausschließlich mit der Hand schrieben, verfassen sie heute viele Text am 
Computer, E-Mails haben die schriftliche Kommunikation verkürzt, SMS-Nachrichten noch mehr. Vielen Schülern falle es daher schwer, längere Texte oder gar ein Buch überhaupt durchzustehen (SPIEGEL, Nr. 2001, S. 64). Flugs wird also mit traditionellem, wenn nicht antiquiertem Gestus verurteilt, was sonst durchaus als lobenswerte Dimensionen von Medienkompetenz geachtet wird - und anderentags womöglich so wieder gefordert wird. So werden, wenn es politisch opportun erscheint, nach wie vor überkommene und zeitgemäße Fähigkeiten gegeneinander ausgespielt.

Noch sicherer gibt sich der notorische Medien-Kritiker des „Vorwärts“, der frühere Planungschef im Kanzleramt unter Helmut Schmidt, Albrecht Müller, der mit seiner altvaterischen Kolumne „Gegen den Strom“ eher unfreiwillig die Paradoxie sozialdemokratischer Medienpolitik zwischen praktischer ungehemmter Deregulierung des Medienmarktes und deshalb unglaubwürdiger Bedenkenträgerei offenbart: „Glotzen macht doof“, titelt er (Müller 2002). Und kurzer Hand behauptet er, dass Vielfernsehen „einen negativen Einfluss auf die Lese- und Lernleistung“ habe. Der PISA-Studie wirft er daher vor, diesen offensichtlichen „Zusammenhang zwischen wachsendem Fernsehkonsum und Schul- und Leseleistung“ nicht geprüft zu haben. Deshalb könne sie auch „nicht als fundierte Entwarnung“ dafür erachtet werden.

Recht hat er nur damit. Denn was nicht Erkenntnisziel und Untersuchungsgegenstand einer zumal empirischen Studie war, kann auch nicht ihr Ergebnis sein. Alles andere ist pure Spekulation, und wieder einmal erweist sich nachhaltig die „Malaise der Medienwirkungsforschung“ (Haff 1998): Was Laien immer wieder fordern oder auch unterstellen, lange Zeit und unermüdlich beim obligatorischen Thema von Mediengewalt und ihren Folgen (Kunczik 1998), nämlich eindeutige Wirkungen zwischen der Rezeption eines Mediums, zumeist des Fernsehens, und erkennbaren Meinungen, Einstellungen und Handlungen zu identifizieren, kann sie so nicht leisten. Allenfalls kann sie in der vorfindlichen Komplexität möglicher Zusammenhänge für diese oder jene Konstellation geringere oder größere Wahrscheinlichkeiten diagnostizieren. Aber diese kasuelle, jeweils valide zu eruierende Probalität können weder eine auf simple Fakten und Kausalitäten erpichte Öffentlichkeit noch schlichte Gemüter begreifen; daher stürzen sie sich auf jede noch so fadenscheinige Beweisführung oder fabulieren sie selbst. Wer in der Medienforschung Publizität erlangen will, braucht nur die eine oder andere, angeblich bewiesene Behauptung über Wirkungsursachen in Umlauf zu bringen, und schon verbreiten es die Medien sensationsheischend (vgl. Kübler 1995a; Kunczik 1998, S.247ff; Winterhoff-Spurk 2001, S. 10ff).

\section{Ergebnisse der PISA-Studie aus medienpädagogischer Sicht}

Könnte PISA nicht auch für die Medienpädagogik nahe legen, was nunmehr für die allgemeine Pädagogik erneut auf der Agenda steht? Denn seit der sogenannten „realistischen Wendung“ (Heinrich Roth) zur (auch empirischen) 
Erziehungswissenschaft Ende der 60er Jahre ist unstrittig, dass Lernen nicht länger nur normativ und in schönen Formulierungen gefordert werden kann und darf, sondern erfolgversprechende Erziehung und Bildung zumal in ihrer wissenschaftlichen Version sich um die realen Bedingungen und subjektiven Möglichkeiten des Lernens kümmern muss. Kann daher nicht ebenso vermutet oder gar befürchtet werden, dass sich bei der spätestens seit 1986 unaufhörlich beschworenen Medienkompetenz (von Rein 1986; Schell 1998) beträchtliche Lücken und Inkonsistenzen auftun könnten, sofern sie vergleichsweise empirisch überprüft würde? Und dies besonders dann, wenn die Zusammenhänge und Interdependenzen untersucht werden, die sie als die vielberufene vierte Kulturtechnik mit den anderen drei ursprünglichen hat oder haben sollte. Deshalb müsste die PISA-Studie auch Medienpädagogen herausfordern, endlich die empirische Substanzen der Medienkompetenz zu ergründen. Doch entsprechende Studien liegen m. W. nicht vor; die wenigen empirischen Erhebungen registrieren vornehmlich institutionelle Vermittlungsangebote, nicht aber individuelle Fähigkeiten und Erfahrungen (Tulodziecki/Schöpf 1992), weshalb in diesem Beitrag nur heuristisch die einschlägigen Dimensionen aufgezeigt und mögliche oder gar erforderliche Erkenntnisse angeführt werden können. Aber insgesamt soll dafür plädiert werden, analog zur Pädagogik auch in der wissenschaftlichen Medienpädagogik die sozialwissenschaftliche Wende zu vollziehen, will heißen: ihre empirischen Desiderate aufzuarbeiten, ihre dahingehenden Aufgaben zu bestärken und die weitgehend erschöpfte Sollensrhetorik offiziellen oder interessierten Instanzen zu überlassen.

Bevor aber nach Zusammenhängen oder gar Ursächlichkeiten zwischen Lesefähigkeit und Medienkompetenz gefragt wird, sollen die dafür relevanten Befunde der sogenannten PISA-Studie (Baumert u.a. 2001) angeführt werden: Trotz der schon angeführten Umorientierung der normativen, kasuistischen Pädagogik zur systematischen, sozialwissenschaftlichen Erziehungswissenschaft sind empirische Befunde über die Lernwirklichkeiten und Lernfähigkeiten der Lernenden offensichtlich spärlich geblieben oder zu wenig zur Kenntnis genommen worden. Insbesondere sind innergesellschaftliche wie erst recht internationale Vergleiche rar. Anders lässt sich das aktuelle Aufmerken über die Leistungen deutscher Schüler nicht erklären. Was unter Eingeweihten längst diskutiert und an unzähligen Fallbeispielen verdeutlicht wurde, das nimmt die (bundesdeutsche) Öffentlichkeit erst erschreckt zur Kenntnis, wenn es mit dem Prädikat großer Daten, mit dem eindeutigen Anspruch von Repräsentativität und wissenschaftlicher Reputation bestätigt wird - und sogleich wird es (bildungs)politisch instrumentiert, wiewohl es sich nur wiederum um eine singuläre, deskriptive Momentaufnahme handelt und die eigentlich aufschlussreichen Verursachungskomplexe nur vermutet, höchstens plausibel nahegelegt werden können.

Trotz aller kompensatorischen bildungspolitischen Anstrengungen, aller Maßnahmen zur Erreichung von Chancengleichheit und verkündeter Förderprogramme sind die Integrations- und Ausgleichleistungen mindestens deut- 
scher Schulen gering geblieben, ja ihre pädagogischen Maßnahmen scheinen sogar soziale Auslese und Ausgrenzungen noch zu verstärken: „Deutschland und die Schweiz gehören zu den Ländern mit den größten Unterschieden in der Lesekompetenz von Jugendlichen aus höheren und niedrigeren Sozialschichten. Die Differenz beträgt in Deutschland mehr als eineinhalb Kompetenzstufen oder 1,2 Standardabweichungen“ (Ebd., S. 40). Oder anders formuliert: In keinem anderen der untersuchten Länder sind die Leistungsunterschiede der Jugendlichen so groß wie hier zu Lande; fast jedes vierte Kind droht damit im bildungspolitischen und damit wohl auch im sozialen Abseits zu landen. Nachdrücklich wird der Schule „bei der Entkoppelung von sozialer Herkunft und dem Erwerb zentraler Basisqualifikationen“ (Ebd.) ein Vorrang, wenn nicht eine exklusive Verantwortung zugeschrieben. Diese Aussage widerspricht gegenteiligen Thesen, die vom Rückgang des schulischen Einflusses und der anhaltende Entschulung der Gesellschaft - nicht zuletzt unter den zunehmenden Wirkungen der Medien - künden. Auch die mehrfach diskutierte Diagnose der wachsenden „Selbstsozialisation“ von Jugendlichen, die wiederum mit dem wachsenden Einfluss von Medien in Zusammenhang gebracht wird (Fromme u.a. 1999), steht konträr zu den konstatierten steigenden Differenzierungen schulischer Leistungen, zumal die egalisierenden Tendenzen des Konsumund Mediensektors entgegen wirken und nivellieren müssten.

In der ersten Phase von PISA sind vorrangig die Lese- und Textkompetenzen (Reading Literacy) 15jähriger Schülerinnen und Schüler überprüft worden. Lesekompetenz wird definiert als die „Fähigkeit, geschriebene Texte unterschiedlicher Art in ihren Aussagen, ihren Absichten und ihrer formalen Struktur zu verstehen und in einen größeren Zusammenhang einordnen zu können sowie in der Lage zu sein, Texte für verschiedene Zwecke sachgerecht zu nutzen“ (Baumert 2001, S. 10). Für sie wurde ein aufwendig differenziertes Konzept mit fünf Kompetenzstufen entwickelt, die von einer elementaren Kompetenz der schlichten Text- und Sinnerfassung bis hin zur Expertenstufe der detaillierten und fundierter Sinnerschließung und -bewertung reicht: „Der Schwierigkeitsgrad einer Aufgabe ist dabei unter anderem abhängig von der Komplexität des Textes, der Vertrautheit der Schülerinnen und Schüler mit dem Thema des Textes, der Deutlichkeit von Hinweisen auf die relevanten Informationen sowie der Anzahl und Auffälligkeit von Elementen, die von den relevanten Informationen ablenken könnten“ (ebd., S. 11). Aus medienpädagogischer Sicht ist ferner zu beachten, dass nicht wie sonst vornehmlich literarische Texte, sondern eine „große Bandbreite an Textsorten“ angewendet wurden: „neben fortlaufend geschriebenen Texten (kontinuierliche Texte), wie zum Beispiele literarische Texte, Argumentationen oder Kommentare [...] auch bildhafte Darstellungen wie Diagramme, Bilder, Karten Tabellen oder Graphiken [...] (nicht-kontinuierliche Texte“) (ebd, S. 10) - mithin Medienprodukte, die von einem umfassenden, elementaren Konzept von Medienkompetenz durchaus als Grundlagen und Befähigungsobjekte erachtet werden könnten. 
Bei diesen Tests erreichten die deutschen Schülerinnen und Schüler nur besagten, so bestürzenden Platz 22 unter 32 Teilnehmernationen und lagen 16 Punkte oder etwa drei Prozent unter dem Durchschnitt. Gegenüber den am besten abschneidenden finnischen Jugendlichen haben die deutschen um fast 12 Prozent schlechtere Leseleistungen. Im Durchschnitt erreichen sie nur eine Lesekompetenz zwischen der II. und III. Stufe von den möglichen fünf. Besonders schlecht schneiden sie beim Reflektieren und Bewerten ab: Hier liegen die deutschen Schülerinnen und Schüler im Durchschnitt 24 Punkte hinter dem Mittel aller 15jährigen. Gravierender noch fallen die Streuungen aus: Hier ist die Differenz zwischen den fünf Prozent leistungsstärksten und den fünf Prozent leistungsschwächsten Schülerinnen und Schüler in Deutschland am größten. Fast zehn Prozent der deutschen Jugendlichen (OECD-Durchschnitt: sechs Prozent) scheitern an den Anforderungen der Kompetenzstufe I, weitere 13 Prozent bewegen sich gerade auf der Kompetenzstufe I. Nach neun, zehn Pflichtschuljahren sind mithin mehr als 20 Prozent deutscher Schüler allenfalls fähig, auf elementarem Niveau zu lesen; selbständiges Lesen und Weiterlernen dürfte dieser „Risikogruppe“ schwer fallen oder ganz aus ihrem Horizont geraten. Aber auch in der Spitze, auf der Kompetenzstufe V, bleiben die deutschen Schülerinnen und Schüler mit fast neun Prozent knapp unter dem OECDMittelwert.

Entgegen allen Prognosen der sozialen Nivellierung oder gar der Individualisierung der Lebensverhältnisse belegen die hier untersuchten Verteilungen der Kompetenzen und Bildungsleistungen „einen straffen Zusammenhang“ mit der zugehörigen sozialen Schicht. In Familien ungelernter Arbeiter steigt der Anteil der schwach Lesefähigen bis auf knapp 40 Prozent an. Diese Entwicklung des Zusammenhangs von sozialer Herkunft und (mangelnder) Leistung erweist sich auch bei Migrationsfamilien, bei denen bereits beide Eltemteile in Deutschland geboren wurde, als immens hartnäckig; er scheint sich immer wieder neu zu bestätigen und zu kumulieren, und zwar bereits vor dem Eintritt in das Schulsystems, ja er verstärkt sich offenbar an jeder Nahtstelle des Bildungssystems. Daher finden sich die typisch schwachen Leser unter den männlichen Jugendlichen aus Migrantenhaushalten oder aus sozial benachteiligten Familien. Mädchen schneiden beim Leseverständnis durchweg besser ab als Jungen, (ebd. S.36).

Ungeachtet möglicher Ursachenerklärung weisen allein diese wenigen Daten auf offensichtliche Diskrepanzen zwischen pädagogisch-didaktischer Theorie, erklärter Bildungspolitik und faktischem, gemessenem Kompetenzstand von Jugendlichen hier zu Lande hin. Allein die Beschwörung einleuchtender, fast selbstverständlicher Ziele und Modelle erzeugt mithin noch keine entsprechende soziale Wirklichkeit. Erneut bestätigt sich eine der grundlegenden pädagogischen Einsichten: dass nämlich Lernen und Können nicht gänzlich und einlinig zu steuern sind, vielmehr dass es von vielen Faktoren und auch Kontingenzen beeinflusst wird, zuallererst von der jeweiligen Persönlichkeit des Lernenden sowie den Optionen und Werten seiner Umwelt. Und in die sind die Medien aufgrund ihrer polyvalenten Symbolstruktur 
vielfach verwoben: als Gegenstände des Lernens selbst (früher als Medienkunde apostrophiert) wie als Mittel und Faktoren des Lernens über alle anderen Sujets (früher: Mediendidaktik).

Warum die Schülerinnen und Schüler eines Landes gute oder weniger gute Leistungen im Lesen erzielen, lässt sich nicht monokausal erklären, sondern hängt von einer Vielzahl von Faktoren ab (ebd., 16). Dafür kann eine empirische Momentaufnahme wie die PISA-Studie nur wenige Anhaltspunkte oder auch Folgerungen liefern. Nur Längsschnittuntersuchungen würden weiterführen. So unterstützt die frühe und drastische Selektion des deutschen Schulsystems eine Förderung gerade Benachteiligter und Förderungsbedürftiger offenbar nicht, die dadurch erfolgte soziale Stigmatisierung und individuelle Demotivation der Ausgelesenen scheinen zu überwiegen. Außerdem ist es in Deutschland mit der Lesebereitschaft und der Leseförderung der Jugendlichen offenbar sehr schlecht bestellt: Kein Land mit vergleichbaren Lesequoten verzeichnet ein höheren Anteil, nämlich 42 Prozent, unter den 15jährigen, die angeben, nicht zum Vergnügen zu lesen. In keiner vergleichbaren Industrienation ergibt sich eine so starke Korrelation zwischen der Zeit, die 15jährigen täglich mit dem Lesen verbringen, und ihren Leistungen. Ob und wie Leseförderung hier zu Lande, in und außerhalb der Schule, betrieben und mit vielfältigen Anreizen forciert wird, untersuchte PISA ebenso wenig gezielt wie Mediengewohnheiten und medienpädagogische Maßnahmen. Solche vergleichende Studien über kulturelle Anregungsoptionen und -milieus fehlen nach wie vor.

Daher hilft es wenig, die Medien und ihre Nutzung gegeneinander auszuspielen. Außerdem belegen Vergleichsdaten etwa der bestplatzierten finnischen Jugendlichen das Gegenteil oder weisen zumindest darauf, dass komplexere Ursachenentdeckungen erforderlich sind: Denn in Finnland sind Computer sowohl in den Schule als auch in den privaten Haushalten ungleich weiter verbreitet, PCs werden im Unterricht schon bei den Jüngsten selbstverständlich, etwa für das Schreiben- und Leselernen, eingesetzt. Dennoch schneiden die finnischen Schülerinnen und Schüler in der Lesekompetenz am besten ab, und der Anteil unter ihnen, der nicht zum Vergnügen liest, ist mit etwa 22 Prozent am geringsten, jedenfalls halb so hoch wie in Deutschland. Leseförderung und Computerschulung müssen sich also nicht ausschließen, vielmehr sind Gewichtungen und wechselseitige Förderungen differenziert aufeinander abzustimmen. Das große Zeitvolumen, das finnische Schülerinnen und Schüler in der Schule verbringen, und ebenso der erhebliche Betreuungsaufwand, den sie auch außerhalb des eigentlichen Unterrichts erfahren, lassen vermuten, dass auf Anleitung und gezieltes Lernen nachdrücklicher Wert gelegt und dem inzidentiellen Selbstlernen außerhalb der Bildungsinstitutionen weniger Bedeutung beigemessen wird. 


\section{Lesefähigkeit und Medienkompetenz - nur wenige empirische Anhaltspunkte} „Abgestumpft und einsam“ - mit solcher Schlagzeile machten Presseberichte - wieder einmal - im Mai 2001 (hier: „Neue Westfalische“ vom 31. Mai 2001) auf eine besorgniserregende Studie von Freiburger Psychologen über die .folgen übermäßigen Fernsehkonsums“aufmerksam, die auch A. Müller (2002) als Beweis dafür anführt, nämlich darauf, dass „die Leseleistung von Schülern [...] mit steigendem Fernsehkonsum ab[nehme]“: Michael Myrtek und Christian Scharff von der Universität Freiburg schockten angeblich mit dem experimentell ermittelten Befund, dass vieles Fernsehen Kinder und Jugendliche emotional abstumpfen und vereinsamen lasse, dass sie ihren Alltag zunehmend als unangenehm und belastend empfinden, dass sie in der Schule versagen, und dies unabhängig von allen sonstigen sozialen Bedingungen und kognitiven Dispositionen. Zwar ist diese Studie schon im Jahr 2000 als Buch - „Fernsehen, Schule und Verhalten“ - publiziert (Myrtek/Scharff 2000; vgl. auch Myrtek u.a. 1997) worden (Gründer 2001), ist also nicht mehr ganz neu. Aber erst im vergangenen Frühjahr wurde sie anlässlich der Verleihung des hochdotierten Wissenschaftspreises „Sicherheit für das Kind“, den ein Komitee von Ärzten und Wissenschaftler unregelmäßig vergibt und den die Winterthur-Versicherung spendiert, öffentlichkeitswirksam popularisiert - mit dem entsprechenden Echo in der Presse (z.B. „Süddeutsche Zeitung“ vom 10. Mai 2001; „Neue Westfälische“ 31. Mai 2001) - was wiederum zeigt, wie sehr die Öffentlichkeit nach solch negativen, möglichst eindeutigen Diagnosen lechzt.

Doch was bedeutet es, 11- bis 15jährige in solch einem Experiment einen Tag und eine Nacht, 23 Stunden lang, an eine tragbares Datenerfassungsgerät anzuschließen, ihre Herzfrequenzen und Bewegungsaktivität zu messen, diese Daten zwischen einer vielsehenden und einer wenigsehenden Gruppe zu unterscheiden und von ihnen auf emotionale Reaktionen und psychische Veränderungen der Jugendlichen durch das Fernsehen zu schließen? Sind auf diese Weise allgemeine und für den Alltag gültige Erkenntnisse zu gewinnen? Erneut müsste daran der Disput zwischen experimenteller, psychologischer und empirischer, soziologischer Forschung geführt werden, er ist freilich in der Medienforschung schon so alt wie diese selbst (Kübler 2000).

Beweisen lassen sich mit solch einem Experiment gewiss keine kausalen Zusammenhänge, erst recht keine langfristigen Wirkungen. Denn ungeprüft bleiben Einflüsse sogenannter Drittvariablen, etwa die soziopsychische Situation jener Jugendlichen, die zur sozialen Isolation, zu einem überdurchschnittlichen Fernsehkonsum, zu sprachlichen und kulturellen Benachteiligungen wie auch zu schlechten Schulleistungen führen kann (und die sich bei PISA umgekehrt in schlechten Schulleistungen niederschlagen). Mithin wäre der postulierte Zusammenhang zwischen Fernsehkonsum, soziopsychischer Deprivation und schulischen Leistungen eine sogenannte „Scheinkorrelation“, weil der Fernsehkonsum zur Verursachung für die genannten Folgen erklärt wird, wohingegen er selbst Folge sein kann. Dabei sei nicht ausge- 
schlossen, dass er seinerseits selbst Folgen zeitigt. Aber welche es sind und in welchem Grad diese Einfluss nehmen, dies lässt sich nicht in einem simplen Experiment klären und nicht in einem nur zweidimensionalen Kausalmodell abbilden. Doch Scheinkorrelationen gerade über die angeblichen Folgen vom Vielsehen sind in der Forschung schon wiederholt aufgedeckt worden, solide Befunden sind hingegen selten; wenn sie vorliegen, ergeben sie keine monokausalen Zusammenhänge (Schulz 1990; Kunczik/Zipfel 2001, S. 406f).

Eine seriösere, freilich zurecht nur als Fallstudie deklarierte Untersuchung über den möglichen Zusammenhang von Lesefähigkeit und Medienkompetenz bei 15jährigen Jugendlichen aus Zürich legten schon in den 80er Jahren die Schweizer Kommunikationsforscher Heinz Bonfadelli und Ulrich Saxer (1986) vor: „Habituelles Lesen kultiviert [...] durch den steten Umgang mit Schrift andere Aspekte als habituelles Fernsehen, und zwar insbesondere darum, weil es mehr eigene mentale Aktivität verlangt und die fixierte Schriftlichkeit des Textes immer schon abstrakt ist, d.h. komplexe analytische, synthetisierende und konkretisierende Verarbeitungsstrategien voraussetzt“ (Bonfadelli 1988, S. 440), postulierten sie, ohne allerdings diese kognitiven Komponenten untersuchen zu können. Gewohnheitsmäßiges Lesen und eine gewisse ausgeprägte Lesekompetenz befähigen dazu, so ihre Schlussfolgerung,, Fernsehsendungen umfassender zu verstehen und mehr Informationen aus ihnen aufzunehmen, also - so man will - eine größere Medienkompetenz zu entwickeln, und zwar unabhängig von dem Grad der formalen Bildung. Habitualisierte Fernsehnutzer, die zudem stärker Unterhaltungssendungen im Fernsehen frequentieren und den unterhaltenden Elementen in allen Sendungen zusprechen, zeigten hingegen ein fragmentiertes Verständnis der Fernsehinhalte und nahmen signifikant weniger Informationen auf. Besonders deutlich erwies sich die Diskrepanz bei den anspruchsvollen und strukturellen Wissensformen. Auch eine österreichische, im Rahmen der Wissenskluft-Perspektive durchgeführte Studie (Fritz 1990), die herausfinden wollte, „ob es die Kulturtechnik ,Lesen ist, die Rezipienten mit kommunikativen Eigenschaften ausstattet, die für die Teilnahme am gesellschaftlichen Kommunikationsprozess und darüber hinaus für gesellschaftliche Lernprozesse notwendig ist“, kommt zu dem Ergebnis: „Es ist wichtig, dass niedriger Gebildete interessiert sind, Bücher lesen, anspruchsvolle Zeitschriften lesen, im Fernsehen und Radio Informationssendungen sehen [und hören, HDK], Informationen suchen und sammeln. All dies bewirkt in seiner Gesamtheit, dass sie Defizite ausgleichen“ (Ebd., S. 130). Stichhaltige und eindeutige Beweise für zwingende Zusammenhänge von Lesen, Verstehen und Medienkompetenz sind dies gewiss nicht; entsprechend vorsichtig oder pauschal sind sie formuliert. Doch die einschlägige Forschung ist auf diesem zentralen Feld jenseits der Zielformulierungen und Behauptungen nicht weitergeführt worden und weitergekommen (Bonfadelli 1999).

So weist etwa Christine Feil (2001a, S. 100) vom Deutschen Jugendinstitut (DJI) zurecht daraufhin, dass die „unterschiedlichen Versuche, Medienkompetenz zu definieren, [...] sich generell durch [eine] erwachsenenzen- 
trierte Perspektive“, orientiert am mündigen, aufgeklärten Bürger, auszeichnen. „Mit ihr kann sicherlich die pädagogische Perspektive der Medienarbeit mit Kindern, d.h. das Erziehungsziel, verdeutlicht werden. Für die Charakterisierung einer kindspezifischen Netzkompetenz, des Verständnisses der Kinder von neuen Technologien, ihrer Probleme und ihres Problemlösungsverhaltens im Internet greift sie zu kurz.“

Dieses Urteil fällt die Autorin nach der Durchsicht der wenigen empirischen Studien über den Umgang von Kindern mit dem Internet (Feil 2001b). Meist begnügen sich diese mit der Reproduktion möglichst beindruckender, aber oft kaum valider Nutzungsdaten, wie sie auch gern in der Öffentlichkeit ventiliert werden. Danach ist die sogenannte (C)-Generationen nicht nur mehrheitlich online, sie geht mit den Optionen des Internets souverän, kompetent und lässig um, und zwar fast schon kurz nach dem Babyalter. Den Eltern und Älteren macht sie jedenfalls alles locker und cool vor, so dass die an den Geräten das Nachsehen haben und unentwegt von ihren versierten Kids lernen müssen. So posaunt es jedenfalls unaufhörlich die öffentliche Legende (z.B. Feibel 2001), auch wenn man über reale Kenntnisse und Fähigkeiten kaum etwas weiß.

Beobachten lässt sich vielmehr, dass Kinder und Jugendliches zwar neugierig und vorbehaltlos an die Geräte herangehen und an ihnen vieles ausprobieren. Das spielerisches handling herrscht vor, trial and error überwiegen. Aber die meisten begnügen sich dann mit relativ simplen Einsichten und Handhabungen, brüsten sich indes gern vor ihren Altersgenossen und den Älteren mit ihrem angeblichen Know-how, das sich oft genug in diese oder jene Kenntnisse über Produkte (Programme), technische Neuerungen und diverse Marken erschöpft. Wenn sich komplexe Aufgaben stellen oder gar Komplikationen ergeben, erlahmt zumeist das forsche, unbekümmert Draufloshacken. Nur wenige Jugendliche machen sich mit Strukturen, Architekturen oder gar Algorithmen der Programme vertraut, die Jungen, so belegen immer wieder fallweise Eindrücke, häufig seltener als die Mädchen, obwohl jene meist mit ihrer Computerkompetenz prahlen. Die Mädchen gehen nicht selten gezielter, funktionsorientierter und überlegter mit den Programmen um, wollen nicht so lange und oft ergebnislos herumspielen, sondern eine Aufgabe erledigen. Deshalb machen sie sich eher mit den grundlegenden Funktionen und Strukturen vertraut (vgl. Turkle 1984; 1999). Ein qualitatives Forschungsprojekt am DJI will nun herausfinden, was Kinder tatsächlich am Computer und mit dem Internet (anfangen) können, auf welche Weise sie sich ihre Fähigkeiten und Erfahrungen aneignen, wo sie reüssieren, wo sie scheitern, wo und wann sie aufgeben, stecken bleiben und wie sie weiterkommen.

Auch unter den durchschnittlichen Erwachsenen entdecken die verfügbaren empirischen Erhebungen entgegen den beliebten öffentlichen Euphemismen nur bei einer sozial, geschlechts-, bildungs- und altersmäßig definierbaren Minderheit den versierten User (ARD/ZDF-Online Studie 2001). Sprichwörtlich wurde schon früher, dass nur wenige ihren Videorecorder angemessen bedienen und all die vorfindlichen, oft auch unnötigen Funktionen erschließen 
können. Die Online-Tätigkeiten beschränken sich ebenso bei den meisten auf wenige Kernfunktionen, die sich zum einen aus evidenten Zwecksetzungen ergeben, zum anderen für das Know-how unaufwändige, zumal nicht mehr in Relation stehende Nutzungskünste erfordern. Nur die sogenannten Technikfreaks erfreuen sich allein an technischen Finessen und befriedigen sich damit, sie nicht selten auf geheimnisvollen, manchmal auch entzweckte Weise zu beherrschen; für die meisten (anderen) sollen sich Alltagsgeräte möglichst evident erschließen und ihre begrenzte Funktionen sicher, unaufwändig und transparent erledigen. Dies gilt auch für den Computer, selbst wenn er zugleich Arbeitsgerät, ästhetisches Gestaltungsinstrument, kommunikatives Medium etc. ist.

\section{Die vierte Kulturtechnik}

Überblickt man - freilich nur noch tendenziell - die unzähligen Positionen, (Definitionsversuche und inhaltliche Ausfüllungen von Medienkompetenz, bleibt sie trotzdem oder gerade deswegen ein disparater, sich ständig verändernder Untersuchungsbereich. Mit der ursprünglichen, aus der Fähigkeit zur sprachlichen Kommunikation (Noam Chomsky) und - später - zum kommunikativen Handeln (Jürgen Habermas) entwickelten Bedeutung haben heute virulente Definitionen und Forderungen nur noch wenig gemein, zumal nicht in den vielen offiziellen Verlautbarungen. Damals ging es darum, die dem Menschen prinzipiell angeborene Sprach- und Kommunikationsfähigkeit auf die technischen Medien zu erweitern, so dass er auch mit ihnen als autonomes, selbstverantwortliches Individuum handeln kann und nicht von irgendwelchen Manipulationsabsichten und ideologischen Beeinflussungsversuchen abhängig wird: Manipulation durch Medien, Aufklärung durch Medienpädagogik bzw. durch ihr Ziel der Medienkompetenz, lautete eine der zentralen Losungen (Schwarz 1976; Schell 1999).

Immerhin weist die Definition als vierte Kulturtechnik auf solch elementare Begründungen und Zusammenhänge hin. Allerdings ist $\mathrm{m}$. W. noch nicht hinreichend geklärt, wie universell diese Kulturtechniken sind, was an ihnen also wie kulturell bedingt ist und was als elementare Prädisposition des Menschen gelten kann, wie dies Wilhelm von Humboldt und in seiner Nachfolge Noam Chomsky postuliert haben. Ebenso müssten die kognitiven (und kommunikativen) Interdependenzen oder gar Prioritäten zwischen den drei ursprünglichen Kulturtechniken eruiert werden. Dabei braucht man nicht mehr von archaischen Zuständen auszugehen, sondern kann die zivilisierten Voraussetzungen und Prägungen bei den Heranwachsenden einbeziehen. Sie wachsen in der Regel in einer durch Schrift und Zahlen strukturierten Umwelt auf, in der Codierung und Decodierung in symbolischen Repräsentanzen, also in Zeichen und Zahlen, zum Grundpensum, zum quasinatürlichen Konstituens des Menschseins gehören.

Natürlich handelt es sich bei der Apostrophierung der Medien- und Computerkompetenz als vierte Kulturtechnik um eine metaphorische Formel; 
in ihrer Substanz will sie indes ernst genommen werden, weshalb man sie daraufhin auch überprüfen sollte. Mit den ersten drei Kulturtechniken sind Fähigkeiten oder auch Techniken der Symbolisierung, der Umsetzung von Wirklichkeitssegmenten - genauer: deren gedankliche Konstrukte - in abstrakte, logische Zeichen- und Zahlensysteme gemeint, die der rationellen und universellen Strukturierung, Quantifizierung, Repräsentanz und Kommunizierung dieser Wirklichkeitsbereiche dienen. Wer die Geschichte der Rechenmaschinen und später des Computers kennt, weiß, dass Mathematik und Arithmetik zu ihren Voraussetzungen zählen (weshalb viele noch heute lieber vom Rechner als vom Computer reden).

Inwieweit und wodurch ist der Umgang mit dem Computer eine spezielle Kulturtechnik, oder ist sie nur die technische Synthese der anderen? Oder bedarf es zwar noch weitere Fähigkeiten, um den Computer angemessen zu nutzen, die zwar in den anderen Kulturtechniken impliziert sind, aber mit ihm nun gesondert und explizit geübt und perfektioniert werden müssen? Etwa die Wahrnehmung und Interpretation von Bildern (die in hiesigen Kulturen meist eine untergeordnete Bedeutung hatten), die Fähigkeit zur logischen Abstraktion, zur strukturierten Deduktion und Gliederung, die Fähigkeit, verschiedene Zeichensysteme aufeinander zu beziehen und ihre speziellen Qualitäten und Funktionen adäquat zu kategorisieren und zuzuordnen etc. Sind solche Dimensionen in jener Rede von der vierte Kulturtechnik mitgedacht, erhält sie ihre Berechtigung. Allerdings müssten sie hinreichend ausdifferenziert und expliziert werden. Vor allem müsste ihre entwicklungsspezifischen und kognitiven Prämissen grundlegend und eigenständig geklärt und empirisch verifiziert werden, um nicht erneut in die technologischen Falle zu geraten. Denn hinsichtlich des Computers wird ja nur apostrophiert und gefordert, was in der jeweiligen Phase technologischer Entwicklung verlangt und beansprucht wird. Und deren Konditionen ändern ja laufend. So sind die rechnerischen und algorithmischen Potenziale des Computers schon weit in den Hintergrund getreten, während die visuellen und so genannten multimedialen Dimensionen immer mehr an Bedeutung gewinnen. Entsprechend ändert sich der kognitiven Umgang: Spielerisches Ausprobieren, trail and error, findiges Durchwursteln sind inzwischen eher gefragt als besonnenes, systematisches Vorgehen. Oft genug findet man sich mit den Widersinnigkeiten, Irrationalitäten und nicht erklärbaren Kuriositäten der Programme und Datenreservoirs ab - und dies bei einer strukturell streng algorithmischen Maschine!

Inhaltliche Dimensionen der Medienkompetenz oder besagter vierter Kulturtechnik haben sich mithin schon mehrfach geändert, und sie werden es weiterhin, wie die Techniken und Maschinen sich wandeln. Wenn der Trend eintrifft, dass die Mikroelektronik zunehmend in andere Gerätschaften zu deren Steuerung integriert werden und zugleich die mikroelektronischen Kommunikationsgeräte sich den menschlichen und alltäglichen Gepflogenheiten anpassen - etwa durch die Verarbeitungsfähigkeit mündlicher Sprache und die Decodierung alltäglicher Aufgaben -, dann dürfte es mit der speziellen Handhabung von Computern, Programmen und Netzen sowie mit der vierten 
Kulturtechnik, soweit sie technisch definiert ist, bald ein Ende haben. So gesehen ist der Computer - wie ursprünglich gedacht - eben nicht etwas Spezielles, sondern die Technifizierung, Automatisierung und Synthetisierung gedanklicher und kommunikativer Operationen (Weizenbaum 1978), und je eleganter und perfekter er diese bewerkstelligt, allerdings jeweils nur in Teilen und separat, umso weniger muss sich der Nutzer um ihre speziellen Prozeduren und technischen Konditionen kümmern.

Macht es daher auf Dauer Sinn, von einer Kulturtechnik zu sprechen, die letztlich jeweils auf den technischen Status Quo bezogen bleibt - auch wenn man sich inzwischen vorderhand bemüht, sie von allzu engen technischen Kautelen zu lösen und sie etwa im Betreich der Wissenserschließung und - beurteilung, im so genannten Wissensmanagement, anzusiedeln? Denn so lange und insoweit sich diese nun apostrophierte Kompetenz wiederum nur auf die kuranten Strukturen und Offerten der digitalen Informationswelt richtet, ist sie zwar eine derzeit notwendige, aber gewiss keine elementare Fähigkeit. Vor allem werden die sie bedingenden kognitiven Fähigkeiten höchstens vordergründig, aber nicht in ihren Substanzen sichtbar. Aber mit der genannten, nun schon erforderlichen Verlagerung hin auf die Evaluation von Information oder gar Wissen - allein diese beiden Phänomene könnten zu neuen explikativen Exkursen provozieren (Kübler 1995b) - zeichnen sich besagte Trends, aber auch neuerliche Dilemmata der theoretischen Bewältigung ab. Denn unweigerlich münden solche Umschreibungen in allgemeine, wenn nicht universelle Dimensionen ein, in die Fragen nämlich, was Menschen heute in einer zunehmend komplexeren, weil mehr und mehr symbolisierten Welt wissen und können müssen, wann und wie sie sich welche Fähigkeiten aneignen, was sie nebenbei und was sie explizit lernen (müssen), wie sie sich sozialisieren und individualisieren. Die digitalen (Kommunikations-)Techniken sind dabei noch immer Vehikel und Erfahrungsobjekte unter anderen; aber welche Bedeutung und welchen Anteil sie einnehmen, ist längst noch nicht hinreichend ermessen und bedürfte aufwändigerer Studien. Just die PISAStudie (und etliche andere Untersuchungen) weisen ja nachdrücklich daraufhin, dass all diese Fähigkeiten und Kenntnisse sozial und kulturell unterschiedlich verbreitet, dass sie mithin von den vorhandenen Bedingungen des Aufwachsens und der Sozialisation beeinflusst, eben nicht egaliter verfügbar und am wenigsten wohl aus den objektiven Konstellationen der technischen Welten deduzierbar sind. Die allfällige Rede vom „Verschwinden der Wirklichkeit“ oder vom „Leben aus zweiter Hand“ verstellt dafür nur den Blick, weil sie pauschal fixiert, was noch offen ist und auch für die erforderliche Selbständigkeit des Individuums mindestens graduell offen bleiben muss. 


\section{Ansätze für eine genuin pädagogische Diskussion über Medienkompetenz}

Einer der wenigen, der auf das doppelte Dilemma der Medienpädagogik hingewiesen hat, ohne es allerdings systematisch zu bearbeiten, war der konservative Pädagoge Rainald Merkert: Die Medienpädagogik habe ein „pädagogisches Defizit“ (Merkert 1992, S. 45ff.), freilich nur weil sie keine pädagogische Theorie habe, mehr noch: weil sie pädagogische Selbstverständlichkeiten ignoriere (ebd., S. 51). Ebenso habe die Pädagogik ein „mediales Defizit“ (ebd., S. 50ff), weil sie Medien lange Zeit missachtete oder nur als Störungen wahrnahm, sie noch heute vielfach als nur kontingente Zusätze, nicht aber als zentrale Momente des Aufwachsens und Erziehens beachtet. Demgegenüber will Merkert von der nach wie vor genuin „pädagogischen Urrelation von Mensch und Welt“ (ebd., S. 56) ausgehen und nach ihren jeweils aktuellen Veränderungen nicht zuletzt oder gerade durch die Medien fragen. Aber aus pädagogischer Sicht bleibt der Mensch im Mittelpunkt des Betrachtens und Bemühens, von ihm geht man aus und zu seiner Entwicklung oder gar Vervollkommnung will man hin. Daher können nicht die Medien, zumal nicht ihre jeweils aktuelle technische Konstitution, ihre gesellschaftliche Organisation und marktkonforme Formierung, vorrangig betrachtet werden (ebd., S. 51). Sonst bleibt die pädagogische Besinnung stets in Abhängigkeit von deren Konjunkturen und Interessen.

So könnte sich - endlich - aus beiden Entwicklungen die Chance, aber auch der Bedarf einer neuen Begründung und Definition von Medienkompetenz (die dann nicht mehr so heißen sollte) ergeben, um zu erkennen, wie Individuen sich in einer technischen Kommunikationswelt zurechtfinden, behaupten oder gar verwirklichen können, mithin welche „Medienkompetenzen“ sich gewissermaßen nebenbei durch ihren Medienkonsum und ihre Medienerfahrungen - also inzidentiell, implizit, kasuell und ungeplant, aber auch selbständig, in eigens intendierten Prozessen des Selbstlernens - ergeben, zumal die Medien entweder erklärtermaßen oder auch als Verkaufs- und Akzeptanzförderung solche Prozesse anstoßen und bestärken, und welche Defizite bestehen (bleiben) sowie welche Medienkompetenzen gezielt, mit professioneller pädagogischer Unterstützung initiiert, gefördert und verstetigt werden müssen. Und eine solche Neubestimmung ergibt sich zum einen aus dem Bestreben, kommunikative Fähigkeiten der Individuen nicht mehr vornehmlich in Abhängigkeit technischer Errungenschaften und medienstruktureller Konditionen zu betrachten, vielmehr eine originär pädagogische Perspektive wieder zu erlangen und das Subjekt ins Zentrum pädagogischer Theorie und pädagogischem Handeln zu stellen - was nicht bedeutet, ahistorische oder atechnische Positionen zu beziehen, vielmehr Optionen von einer autonomen Warte zu beurteilen und ihre erforderlichen Veränderungen anzumahnen, sowie zum anderen aus den erkennbaren Entwicklungstendenzen, dass sich - wie gesagt - die Medien(geräte) zunehmend den menschlichen Gepflogenheiten annähern, also humaner und reflexiver wer- 
den, mithin man nicht mehr ihre technische, reichlich abstrakte Logik bei ihrem Gebrauch nachvollziehen muss. Dazu sollen einige Dimensionen freilich nur noch mit heuristischem Anspruch aufgeführt werden:

\subsection{Mediale Einflüssen auf das Aufwüchsen (Entwicklung) und Sozialisation}

Noch immer formulieren Entwicklungspsychologie und Pädagogik ihre Dynamiken und Modelle menschlicher Entwicklung weitgehend ohne Berücksichtigung medialer Faktoren, ungeachtet der Thesen über Akzeleration und „Selbstsozialisation“ durch Medien; allenfalls werden diese Faktoren als Behinderungen und Störungen, nicht als unweigerliche Momente heutigen Aufwachsens und gegenwärtiger Sozialisation erachtet. Umgekehrt müsste sich medienpädagogische Forschung um die grundlegenden kognitiven wie perzeptiv-rezeptiven Fähigkeiten von Kündern und Jugendlichen kümmern, und zwar sowohl differenziert nach den sozialen Milieus, kulturell-ethnischen Besonderheiten und sonstiger Einflusskonstellationen als auch im Gesamt diverser medialer Tätigkeiten - vom Lesen bis zur Internet-Nutzung - und ihrer möglichen wechselseitigen Beeinflussung.

Wenn es die Kinder und die Jugend nicht mehr gibt, sondern vielfältige Milieus, Szenen, Soziotopen (z.B. Hitzler u.a. 2001) - und wie die Differenzierungstermini alle heißen -, dann finden sich diese nicht nur in diversen Mediennutzungsmustern, sondern auch in unterschiedlichen (De)Codierungsfähigkeiten von Kindern und Jugendlichen wieder - worauf die PISA-Studie hinweist. Folglich lassen sich diese nicht mehr allein nach Alter bzw. deren einschlägigen Konstrukte formulieren, sondern müssten entsprechend differenziert werden: nach soziale Bedingungen, kulturellen Einflüssen, geschlechtsspezifische Unterschieden. Nehmen zum Beispiel türkische Mädchen mediale Bilder anders wahr als gleichaltrige deutsche oder auch als türkische Jungen, und worin unterscheiden sie diese Wahrnehmungen? Kann extensiver Medienkonsum wirklich als Faktor der Entwicklungsbeschleunigung (oder auch -hemmung) identifiziert werden, oder ist er bereits Produkt grundlegenderer sozialer Konstellationen, die ihrerseits kognitive, kommunikative und perzeptive Entwicklungen prägen? Fragen dieser Art lassen sich vielfältig stellen; sie signalisieren damit den nach wie vor ungelösten Forschungsbedarf.

\subsection{Weltwahrnehmung, Perzeption und Realitätssinn}

In welchen Welten leben Kinder und Jugendliche heute? Auf die pauschalen Verdikte über das Überhandnehmen zweiter Realitäten ist bereits hingewiesen worden. Allfällige Klagen über mangelnden Realitätssinn von Kindern und Jugendlichen und über ihre Neigungen, sich vorrangig an Medienidolen und Konsummarken zu orientieren, häufen sich in populären Publikationen. 
Andererseits wird immer wieder erstaunt festgestellt, dass Kinder und Jugendliche recht pragmatisch und selbstverständlich mit all den überbordenden Attraktionen und Faszinosa umgehen, die ihnen die einschlägig professionellen Erwachsenen anbieten oder aufdrängen, und mit ihnen oder auch gegen sie durchaus ihren Eigensinn und ihre Identität entwickeln.

So kommt ja die Soziologie (z.B. Beck 1986) zu ihrer These der wachsenden Individualisierung von Lebensläufen zumindest in traditioneller und formeller Hinsicht, während neue Standardisierung auf symbolischen Ebenen - nicht zuletzt durch Medien und ihre konformen Netzwerke - drohen. Doch welchen Stellenwert haben diese gegenüber der Herauslösung der Biographien aus den „unmittelbaren Lebenskreisen“ (ebd, S. 219)? Sind etwa zu beobachtende Neigungen wachsender ,performances‘ des Ichs in jedweder Fasson Antworten auf diese Widersprüche - so nach der Devise: In der Substanz richtet man sich nach Normalität, im zeitbedingten, situationsbedingten outfit erprobt man andere, grelle Identitätsentwürfe? Viele der Jugendstudien weisen in diese Richtung; was sie indes als empirische Momentaufnahmen noch kaum eingelöst haben, sind längerfristige Dynamiken und Übergänge, Kontinuitäten und Diskontinuitäten in der Entwicklung und Sozialisation auszumachen. Immerhin könnte das Aushalten oder sogar das bewusste Demonstrieren solcher Inkonsistenzen auf beachtliche Sozialisations- und Integrationsleistungen der Individuen hindeuten, die überkommene Postulate vom Streben nach innerer Konsonanz und Harmonie, wie sie nicht nur in der Kommunikationswissenschaften (vgl. Kunczik/Zipfel 2001, S. 308ff), sondern eben auch in den pädagogischen Idealen einer Persönlichkeit hochgeschätzt wurden und werden, überholen könnten.

\subsection{Kognition und Wissen}

Seit über allgemeine Fragen der Bildung hierzulande wieder nachdrücklicher gestritten wird, stellen sich auch immer wieder Fragen nach den kognitiven Fähigkeiten und dem Wissen von Kindern und Jugendlichen. Allerdings werden diese eher normativ - im Sinne überkommener Wissenskanones beantwortet - selten empirisch (z.B. Elschenbroich 2001). Statt konkret und kontinuierlich zu fragen, was Kinder heute können und wissen, woher sie ihr Wissen beziehen, ob eher aus realen, sinnlichen Erfahrungen, eher aus symbolischen, medialen Welten oder infolge eigenständiger, individueller Mixturen, und danach, wie sich ihre Wissenskontingente sozial und kulturell verteilen, verlocken eher Konzepte, die Wissens- und Kompetenznormen umreißen und pädagogische Empfehlungen ihrer Erreichbarkeit formulieren. Anders lässt sich der unerwartete Bestsellererfolg von D. Elschenbroichs Entwurf (12. Aufl. 2002) nicht erklären.

So bleibt bis auf weiteres die Vermutung unbelegt, dass Kinder heute mehr und komplexere Dinge können und wissen müssen als in früheren Phasen. Konstruiert werden sie bislang nur aus Beobachtungen der vielen für 
Kinder relevanten bzw. sie faszinierenden Dinge ihrer Umwelt. Vergleichende historische Studien wären nur aufwändig und mit einigen methodischen Heuristiken zu bewerkstelligen, da für vergangene Wissensstadien auf mehr oder weniger aufschlussreiche Quellen zurückgegriffen werden müsste. So stehen sich heute zwei pauschale Thesen gegenüber, die eine, die das über Hand nehmende Leben aus zweiter Hand beklagt, und die andere, die sich an der immer wieder zu beobachtenden Lebenstüchtigkeit der Kinder erfreut, an ihren immer wieder verblüffenden Fähigkeiten, sich in einer ständig unübersichtlicher und widersprüchlicher Welt zurechtzufinden.

\subsection{Fiction, Spaß und Escape}

Dass Kinder und Jugendliche fast ausschließlich Spaß und Ablenkung haben und sich kaum mehr für ernste, seriöse Angelegenheiten engagieren wollen, gehört ebenfalls zu den notorischen Klagen (und passt nicht ganz ins vornehmlich kognitive Ziel der Medienkompetenz). Denn Spaß haben lässt sich auch ohne große kognitive Anstrengung ermöglichen, selbst wenn der am klassischen Ideal geschulte Geist primär elaborierte Niveaus von Amüsement unterstellt. Aber die bringt er kaum mehr mit den gängigen Medien in Verbindung, wo fast nur noch Klamauk, Flachsinn oder gar Trash obsiegen. Sind im üblichen Zielhorizont „Medienkompetenz“ überhaupt noch solche Komponenten von sinnlichem Vergnügen, emotionaler Empathie und ästhetischer Befriedigung mitgedacht? Ursprünglich mitunter schon, auch wenn nie ausreichend expliziert und ausdifferenziert. Die Konzentration oder gar Reduktion auf einen (engen) Begriff kritischer Vernunft war stets beherrschend und damit dem vorrangig auf Fiction, Spaß und Escape fixierten Mediensystem kaum angemessen. Doch mit der Ausformulierung theoretischer Konzepte wie pädagogischer Ideale von Phantasie und Vergnügen tut man sich seit jeher schwer; über das strenge klassische Junktim von „prodesse et delectare“ sind sie kaum hinausgelangt. Wenn neuerdings auf Informations- und Wissenskompetenz ab-gehoben wird, so können unter einem abstrakten Begriff von Information auch jene Bereiche mitgedacht werden, im gängigen Sprachgebrauch stehen sich Unterhaltung und Information jedoch konträr gegenüber Deshalb hat sich die Medien- und Konsumbranche auf das verbale Kunstmix von Infotainment und Edutainment verständigt, um sich so von den ernsthaften Kautelen zu lösen und mit ihren Attraktions- und Zerstreuungsstrategien machen zu können, was sie will. Dem seriösen pädagogischen Anliegen bleiben solche Bestrebungen suspekt, weil Lernen nicht gleichsam nebenbei, bequem und light gelingt, sondern Anstrengung, Ausdauer und auch Frustration(stoleranz) einschließen muss - allerdings nur unter einem pädagogischen Lernbegriff, nicht unter einem behavoristischen. Wie aber vermittelt man solches Know-how, zwischen verschiedenen Lernformen zu unterscheiden, die jeweils angemessene zu wählen, nicht ausschließlich den amüsanten Verführungen der elektronischen Lernindustrie zu verfallen, aber sie 
auch nicht a priori zu auszuschließen und abzuwerten, weil sie sich sinnlicher und lustiger präsentieren?

Hört man die eine Seite, überwiegen die Klagen darüber, dass Kinder und Jugendliche heute infolge des medialen Dauerbombardements nicht mehr imstande seien, konzentriert, systematisch und ausdauernd zu lernen. Folgt man der anderen, dann sind solch dröge, anstrengende Lernmethoden einfach nicht mehr zeitgemäß, weil medial überholt und ineffektiv. Verbindungen mit den Inhalten werden indes kaum gezogen, und Fragen, was sich wie lernen lässt, werden nicht differenziert genug untersucht.

\subsection{Konsumismus}

Legio sind auch die Monita, dass Kinder und Jugendliche ausgeprägte konsumtive Neigungen bis hin zu bedrohliche Manien für Kauf und Verschwendung haben, dass sie ein nachhaltiges, demonstratives Markenbewusstsein besitzen, über das sie nicht zuletzt soziales Prestige in der Gleichaltrigengruppe und persönliche Identität definieren, dass sie Geld leichtsinnig bis hin zu wachsenden Schulden verschwenden und jeden Modeschrei mitmachen, kurzum von einer konsumistischen Grundstimmung durchsetzt sind, wonach alles käuflich ist, zum Konsum und Konsum gereichen muss. Wäre diese Neigungen so massiv, durchgängig und folgenreich, wundert es, warum Konsum, Werbung und finanzielle Verantwortlichkeit mindestens in der institutionellen Erziehung und Bildung eine vergleichsweise geringe Bedeutung haben (Deutsches Jugendinstitut 1997). Auf der anderen Seite mehren und verstärken sich die Marketing- und Werbeanstrengungen, Kinder und Jugendliche noch enger mit Verkauf und Konsum zu verstricken, ihnen noch aggressiver und wirksamer die Disposition einzuimpfen, ihre Identität und die anderer nach dem Besitz von Waren, nach Markenkenntnissen und nach der Option zu taxieren, wie viel Geld für Events, Freizeit und Konsum verschwendet werden kann. Und es sind ebenso Erwachsene, oftmals die in der Gesellschaft erfolgreicheren und verwöhnteren, die ihnen diese Strategien und Versprechungen andienen, ebenso wie es Erwachsene sind, die sie davor bewahren wollen, ihnen kritisches Bewusstsein oder wenigstens gewisse Distanz und Reflexionsfähigkeit vermitteln wollen. Auch mit diesen Konkurrenzen müssen Kinder und Jugendliche heute aufwachsen, sie müssen sie buchstäblich aushalten und durch sie hindurch ihre Persönlichkeit entfalten, ihre Bedürfnisse und ihre Möglichkeiten herausfinden und einigermaßen authentisch vertreten.

In den wenigen empirischen Studien über die Einsicht und Resistenz von Kindern und Jugendlichen gegenüber Werbung (Charlton u.a. 1995; Baacke u.a. 1999) wird erstaunt bis erfreut konstatiert, dass Kinder mit zunehmenden Alter eine deutliche Distanz, wenn nicht Abneigung gegen Werbung entwickeln und deren Absicht recht klar durchschauen: Nur jüngere Kinder zwischen vier und sieben Jahre mögen Werbung, allerdings nur weniger als die Hälfte. Bei den älteren Kindern sind es deutlich weniger, bei den 11- bis 
14jährigen gerade noch gut ein Prozent (Charlton u.a. 1995, Bd. 2, S. 266). Je älter die Kinder sind, desto kritischer werden sie gegenüber der Werbung. Befragungen zum Item „Werbekompetenz“ fuhren dennoch zu dem Resümee, dass „nur eine sehr kleine Minderheit von Kindern unter 14 Jahren die Bedeutung der Werbung für das eigene Kauverhalten umfassend erkennen kann“ (ebd., S. 265). Wiederum tut sich also eine Diskrepanz auf zwischen verschiedenen Wissens- und Urteilsdimensionen, zwischen kognitiven und emotionalen Strebungen; aber sie dürfte nicht zuletzt auch den entsprechenden normativen Ansprüchen der Untersuchenden geschuldet sein. Solche Klüfte bestätigen sich auch darin, dass Kinder und Jugendliche zwar Werbung nicht gerade favorisieren, aber in ihrem alläglichen Verhalten und ihren normativen Orientierungen durchaus konsumistische Neigungen und Werte erkennen lassen, wie zumindest die landläufigen Klagen hervorheben. Dabei sind bislang vorwiegend nur altersspezifische Entwicklungen, Mädchen und Jungen, aber kaum noch soziale, kulturelle und ethnische Differenzen in den Dispositionen unterschieden worden, wie wiederum die PISA-Studie auch für den Umgang mit Werbung, für Werbekompetenz und konsumistische Mentalitäten nahe legen würde. Denn die Spannweite, die Kinder und Jugendliche auch hierbei wie bei anderen Orientierungen zeigen, reicht heute auch noch von der demonstrativen oder lässigen Konsumaskese bis hin zum kompletten Styling nach und bis hin zur schon manischen Unterwerfung unter die jeweils aktuellen Modetrends und Konsumhits. Werbewirkungen und Einstellungen zu Werbung müssen heute mithin komplexer, gründlicher und langfristiger untersucht werden, als es die üblichen psychologischen Tests vorsehen (Winterhoff-Spurk 2001, 83ff).

Dies alles sind nur heuristischen Skizzen darüber, wie weit, wie gründlich und wie langfristig Dimensionen von Medienkompetenz reichen (müssen), sofern ihr vielbemühter Anspruch nicht nur deklamiert wird, sondern für den Alltag und für die Subjekte relevant untersucht und beschrieben wird. Sicherlich rechnen noch einige weitere Segmente dazu. Vor allem aber müssten sie auf die elementaren Entwicklungsbereiche und Fähigkeiten bezogen, deren Interdependenzen und wechselseitigen Dynamiken ausgelotet und nicht gegeneinander ausgespielt werden. Aufwachsen heute passiert nicht mehr ohne Medien und Konsum, ebenso wenig wie auf besagte elementare Kulturtechniken verzichtet werden kann. Aber diese nur auf traditionelle Weise zu beschwören und sie unentwegt durch Technik, Konsum und Medien bedroht sehen, hilft ebenso wenig, wie von den neuen Medien quasi automatisch ihre Wiederbelebung und Förderung zu erwarten. Das hatte bereits der berühmte Nürnberger Trichter nicht vermocht, und alle überzogenen Erwartungen an Lerntechnologien danach mussten über kurz oder lang ihr Scheitern oder zumindest ihre nur begrenzte Wirksamkeit einräumen. So wird es auch den Beschwörungen des Internet als die Chance und Herausforderung einer neuen Bildung ergehen. Denn entscheidend und (für sich) verantwortlich bleibt das Individuum, an ihm müssen sich alle Lernstrategien abarbeiten, durch es werden sie gewissermaßen individuell gebrochen und neu konstruiert. Und da mit der Medienexplosion 
die für alle Individuen verfügbaren symbolischen Welten - die zugleich, jenseits jeder Wertung, stets auch kognitive Lernwelten sind - enorm zunehmen, wachsen die Optionen des Selbstlernens unentwegt, verlieren ehemalige Dominanzen oder gar Monopole des formalen Lernens wie die Schule ihren überragenden Einfluss, müssen sich zumindest mit anderen Lernkonkurrenzen auseinandersetzen und gegen sie behaupten.

Daher kann nur noch einmal dafür plädiert werden, Medienkompetenz in der umrissenen Vielfalt wie auch in der empirischen Diversifikation bei den Individuen zu untersuchen und sie nicht länger nur normativ zu postulieren. Fragen, wie Kinder und Jugendlichen divergierender sozialer, kultureller und ethnischer Provenienzen in verschiedenen Altersphasen unterschiedlich wahrnehmen, Figuren und Dramaturgien interpretieren, was sie emotional anspricht und was nicht, was sie berührt und was sie „cool“ lässt, wie sie sich ihre Wissen zusammenkonstruieren, aus den Medien, aber auch außerhalb von ihnen, wie sie sich Vor- und ihre Weltbilder basteln u. v .a .m - all das sind empirisch zu eruierende Momente, die in Zielformulierungen von Medienkompetenz einfließen müssten. Und wenn diese - nicht nur im Einklang mit den (medientechnischen Entwicklungen - weiter als kommunikative Kompetenz gefasst wird, dann erweitern sich natürlich auch die einzubeziehenden Dimensionen auf die menschliche Artikulation und Kommunikation allgemein - nicht nur auf die Fähigkeit, SMS zu verschicken -, auf ästhetische Fähigkeiten, auf kognitive Kompetenzen wie auf soziale Empathie, die Kinder und Jugendliche jeweils mit Medien, vor ihnen und auch über sie hinaus entwickeln.

\section{Literatur}

ARD/ZDF-Online Studie 2001. In: Media Perspektiven, H. 8,2001.

Baacke, D. u.a. : Zielgruppe Kind. Kindliche Lebenswelten und Werbeinszenierungen. Opladen 1999.

Baumert, J. u. a.: OECD - PISA. Programme for International Student Assessment. Schülerleistungen im internationalen Vergleich. Zusammenfassung zentraler Befunde 2001 (http:://www.mpibberlin.mpg.de/pisa/pdfs/ergebnisse.pdf).

Beck, U.: Risikogesellschaft. Auf dem Weg in eine andere Moderne. Frankfurt/M. 1986.

Bonfadelli, H.: Lesen, Fernsehen und Lernen. Eine Studie über differentielle Medieneffekte bei 15jährigen Zürcher Jugendlichen. In: Publizistik, 33 (1988) 2-3, S. 437-455.

Bonfadelli, H.: Die Wissenskluft-Perspeküve. Massenmedien und gesellschaftliche Information. Konstanz 1994.

Bonfadelli, H.: Leser und Lese verhalten heute - Sozial wissenschaftliche Buchlese(r)forschung. In: Franzmann, B. u.a. (Hrsg.): Handbuch Lesen. München 1999, S. 86-144.

Bonfadelli, H./Saxer, U.: Lesen und Fernsehen. Zug 1986.

Charlton, M. u.a.: Fernsehwerbung und Kinder. 2 Bde. Opladen 1995.

Deutsches Jugendinstitut (Hrsg.): Werbepädagogik in der Grundschule. Opladen 1997.

Elschenbroich, D.: Weltwissen der Siebenjährigen. Wie Kinder die Welt entdecken können. München 2001.

Feibel, Th.: Die Internet-Generation. Wie wir von unseren Computern gefressen werden. Berlin 2001. 
Feil, Ch. (a): E-Mails und Chaträume ...für Kinder? In: Palme, H.-J./Natasa, B. (Hrsg.): a.a.0.2001, S. 95-103.

Feil, Ch. (Hrsg.) (b): Internet für Kinder. Hilfen für Eltern, Erzieher und Lehrer. Opladen 2001.

Fritz, A.: Individuelle Kommunikationsstrukturen. Information und Wissen. Wien 1990.

Fromme, J. u.a. (Hrsg.): Selbstsozialisation, Kinderkultur und Mediennutzung. Opladen 1999.

Gründer, H.-U.: Fernsehen, Schule und Verhalten. In: medien praktisch, 25. Jg., 2001, H. 2, S. 68.

Halff, G.: Die Malaise der Medienwirkungsforschung: Transklassische Wirkungen und klassische Forschung. Opladen 1998.

Hitzler, R. u.a.: Leben in Szenen. Formen jugendlicher Vergemeinschaftung heute. Opladen 2001.

Kübler, H.-D.: Mediengewalt: Sozialer Ernstfall oder medienpolitischer Spielball? Ein Dauerthema im Interessenclinch zwischen Politik, Kommerz und Wissenschaft In: Friedrichsen, M/Vowe, G. (Hrsg.): Gewaltdarstellung in den Medien. Theorien, Fakten und Analysen. Opladen 1995a, S. 69-108.

Kübler, H.-D.: Zauberwort „Information“. In: Fachbereich Bibliothek und Information der Fachhochschule Hamburg (Hrsg.): Biblionota. 50 Jahre bibliothekarische Ausbildung in Hamburg 25 Jahre Jahre Bibliothek und Information. Münster und New York 1995b, S. 225-254.

Kübler, H.-D.: Mediale Kommunikation. Tübingen 2000.

Kübler, H.-D.: Leaming by Surfing? Digitale Lernmythen und Wissensillusionen. In: Bürdek, B. (Hrsg.): Der digitale Wahn. Frankfurt/M. 2001a, S. 147- 77.

Kübler, H.-D.: Neue Medien - neues Lernen? Perspektiven und Bedingungen des schulischen Lernens mit „neuen Medien“. In: Felsmann, K.-D. (Hrsg.): 4. Buckower Mediengespräche: Neue Medien - neues Lernen? München 2001b, S. 17-36.

Kunczik, M. Gewalt und Wirkungen. 4. aktualisierte Aufl. Köln u.a. 1998.

Kunczik, M./Zipfel, A.: Publizistik. Köln u.a. 2001.

Merkert, R.: Medien und Erziehung. Einführung in pädagogische Fragen des Medienzeitalters. Darmstadt 1992.

Müller, A.: Glotzen macht doof. Wer Bildungsreform fordert, muss auch über das Fernsehen reden. In: Vorwärts 2/2002, S.l 1.

Myrtek, M. u.a.: Psychophysiologische Untersuchungen zum Fernsehverhalten bei 11- bis 15jährigen Schülern unter besonderer Berücksichtigung der emotionalen Reaktionen. In: Charlton, M./ Schneider, S. (Hrsg.): Rezeptionsforschung: Theorien und Untersuchungen zum Umgang mit Massenmedien. Opladen 1997, S. 122-146.

Myrtek, M./Scharff, Ch. : Fernsehen, Schule und Verhalten. Untersuchungen zur emotionalen Beanspruchung von Schülern. Göttingen u.a. 2000.

Palme, H.-J./Natasa B. (Hrsg.): Medienkompetenz. Version 2002. Navigationshilfen für Kinder, Jugendliche und Erziehende. Bielefeld 2001.

Schell, F./Stolzenburg, E./Theunert, H. (Hrsg.): Medienkompetenz. Grundlagen und pädagogisches Handeln. München 1999.

Schiersmann, Ch. u.a.: Medienkompetenz - Kompetenz für neue Medien. Studie und Workshop. Bonn 2002 (Materialien des Forum Bildung).

Schulz, W.: Fernseh-Paranoia und andere psychische Auffälligkeiten: Langzeitwirkungen des Fernsehens? In: Kunczik, M/Weber, U. (Hrsg.): Fernsehen. Aspekte eines Mediums. Köln/Wien 1990, S. $112-120$.

Schwarz, R. (Hrsg.): Manipulation durch Massenmedien - Aufklärung durch Schule? Stuttgart 1974 (Didaktik der Massenkommunikation 1). 
Tulodziecki, G./Schöpf, K.: Zur Situation der schulischen Medienpädagogik in Deutschland: Konzepte, Materialien, Praxis und Probleme. In: Bertelsmann Stiftung (Hrsg.): Medienkompetenz als Herausforderung an Schule und Bildung. Gütersloh 1992, S. 104-176.

Turkle, S.: Die Wunschmaschine. Der Computer als zweites Ich. Hamburg 1984.

Turkle, S.: Leben im Netz. Identität in Zeiten des Internet. Hamburg. 1999.

Weizenbaum, J.: Die Macht der Computer und die Ohnmacht der Vernunft. Frankfurt/M. 1978

Winterhoff-Spurk, P.: Fernsehen. Fakten zur Medienwirkung. 2., völlig Uberarb. u. erg. Aufl. Bern u.a. 2001. 